\title{
Estoque doméstico e uso de medicamentos em uma população cadastrada na estratégia saúde da família no Brasil ${ }^{1}$
}

\author{
Patricia de Carvalho Mastroianni, ${ }^{2}$ Rosa Camila Lucchetta, ${ }^{3}$ \\ Josiane dos Reis Sarra ${ }^{3}$ e José Carlos Fernandez Galduróz ${ }^{4}$
}

Como citar Mastroianni PC, Lucchetta RC, Sarra JR, Galduróz JCF. Estoque doméstico e uso de medicamentos em uma população cadastrada na estratégia saúde da família no Brasil. Rev Panam Salud Publica. 2011; 29(5):358-64.

RESUMO Objetivo. Identificar domicílios atendidos pela estratégia saúde da familia (ESF) que possuíam estoque de medicamentos, avaliar as condições de armazenamento e conhecer o modo de uso dos medicamentos.

Métodos. O estudo foi conduzido em um município do Estado de São Paulo que possui duas unidades da ESF, com 1867 domicilios cadastrados; a amostra foi definida por meio de sorteio aleatório estratificado. A coleta de dados foi realizada por meio de entrevistas semiestruturadas de julho a outubro de 2008.

Resultados. Foi entrevistado um morador em cada um dos 280 domicilios visitados. Foram encontrados medicamentos em 255 domicílios (91,1\%). Dos 326 locais de guarda de medicamentos, $217(75,8 \%)$ eram inadequados (de fácil acesso a crianças ou expostos a umidade, luz). Das 2578 especialidades farmacêuticas encontradas, 2059 (79,9\%) tinham algum problema de segurança ou identificação, o que foi observado em $236(84,3 \%)$ domicílios. Dos 280 entrevistados, 179 (63,9\%) eram usuários de medicamentos. Desses, 24 estavam se automedicando, apenas um com medicamento que não exigia prescrição. Somente 44 usuários tinham a prescrição do medicamento. O medicamento era usado de forma diferente da prescrição por 21 entrevistados, por desacordo posológico ou interrupção do tratamento.

Conclusões. A maioria dos domicílios estudados armazenava os medicamentos de forma inadequada. As discordâncias em relação às orientações médicas prescritas podem levar a resultados negativos, como ineficácia por uso de doses abaixo do prescrito, intoxicações por doses acima do prescrito, reações adversas e não adesão terapêutica.

Palavras-chave Assistência farmacêutica; Programa Saúde da Família; uso de medicamentos; armazenagem de medicamentos; automedicação; Brasil.

Estudo apresentado no Simpósio Internacional de Atenção Farmacêutica (Universidade Federal de Alfenas, Minas Gerais, 14 a 16 de maio de 2009); na Conferencia Iberoamericana de Facultades de Farmacia (COIFFA) - III Congreso de Ciencias Farmacéuticas (Universidad Privada Antonio Guillermo Urrelo, Cajamarca, Peru, 30 de maio de 2009); no VII Congresso Brasileiro de Farmácia Hospitalar - Sbrafh (Minascentro, Belo Horizonte, Minas Gerais, 13 de junho de 2009); e na Cerimônia do Prêmio Nacional de Incentivo à Promoção do Uso Racional de Medicamentos (Ministério da Saúde, Brasília, Distrito Federal, 14 de dezembro de 2009).
A Organização Mundial da Saúde (OMS) e, no Brasil, a política nacional de medicamentos $(1,2)$ preconizam que o

2 Universidade Estadual Paulista (UNESP), Faculdade de Ciências Farmacêuticas, Departamento de Fármacos e Medicamentos, Araraquara (SP), Brasil. Correspondência: pmastro@fcfar.unesp.br

3 UNESP, Faculdade de Ciências Farmacêuticas, Departamento de Fármacos e Medicamentos, Araraquara (SP), Brasil.

4 Universidade Federal de São Paulo (UNIFESP), Departamento de Psicologia, São Paulo (SP), Brasil. uso racional de medicamentos se dá quando os pacientes recebem a medicação adequada às suas necessidades clínicas, nas doses correspondentes aos seus requisitos individuais, durante um período de tempo adequado e ao menor custo possível para si e para a comunidade. Já a automedicação é definida como o uso de medicamento sem prescrição, orientação ou acompanhamento do médico ou do dentista (3). Considerando-se 
que $35 \%$ dos medicamentos são adquiridos no Brasil para automedicação (4) e que, desses, 44,1\% necessitam da apresentação da prescrição médica para aquisição, observa-se que a automedicação, na maioria das vezes, é inadequada, podendo trazer prejuízo ao usuário (5).

Outra prática muitas vezes inadequada é a "polifarmácia doméstica" estoques domiciliares de medicamentos sujeitos a prescrição médica ou a controle especial que sobram de tratamentos anteriores ou são adquiridos sem a devida prescrição, ou ainda medicamentos isentos de prescrição. No domicílio, além do problema intrínseco da facilidade de acesso a medicamentos sem orientação médica, pode haver problemas de armazenamento: os medicamentos devem ser guardados em local arejado e seguro, sem exposição à luz, calor ou umidade, em sua embalagem original, identificados pelo nome comercial ou genérico e princípio ativo e com data de validade e lote, o que nem sempre ocorre.

Segundo Margonato et al. (6), a população estoca medicamentos em casa devido à inconstância na sua disponibilidade nas unidades básicas de saúde (UBS), ou ainda por ter medo de ser acometida por doenças e porque desconhece os riscos que os medicamentos podem acarretar se não forem armazenados corretamente. Em consequência, são inúmeros os casos de medicamentos vencidos e de intoxicações medicamentosas. Segundo dados do Sistema Nacional de Informações Tóxico-Farmacológicas, os medicamentos ocupam a primeira posição entre os três principais agentes causadores de intoxicações em seres humanos desde 1996, sendo que, em 2007, foram responsáveis por 30,3\% dos casos registrados no Brasil (7).

Nessa situação, sabe-se que o uso inadequado de medicamentos onera os serviços de saúde com atendimentos, internações e óbitos $(4,8)$. Pode provocar agravamento do quadro clínico, enfermidades iatrogênicas, interações, reações adversas a medicamentos e resultados negativos associados à medicação (9-11). Estima-se que cerca de $15 \%$ das internações hospitalares sejam por reações adversas a medicamentos (12) e que $92 \%$ dos casos de problemas relacionados com medicamentos no âmbito secundário e terciário da saúde possam ser prevenidos (13).

No âmbito da atenção primária à saúde, espera-se que iniciativas como a estratégia saúde da família (ESF) possam identificar e prevenir os resultados negativos associados à medicação, bem como diminuir os gastos hospitalares pelo uso inadequado de medicamentos. Portanto, o presente estudo teve como objetivo descrever a ocorrência de estoques de medicamentos nos domicílios, as condições de armazenamento e o modo de uso desses medicamentos em uma população atendida pela ESF no Estado de São Paulo.

\section{MATERIAIS E MÉTODOS}

Trata-se de um estudo farmacoepidemiológico do tipo transversal, descritivo e observacional, conduzido num município que se encontra a $360 \mathrm{~km}$ da capital paulista. Sua atividade econômica predominante é a agropecuária. A população é de aproximadamente 14 mil habitantes (14). O município apresenta cinco serviços de saúde ambulatoriais, sendo um privado, e duas unidades da ESF.

$\mathrm{O}$ universo da pesquisa correspondeu aos 1867 domicílios cadastrados nas duas unidades da ESF em 2008, sendo 809 na ESF-1 e 1058 na ESF-2. A amostra foi definida utilizando-se o cálculo de amostragem de proporção, com intervalo de confiança de $95 \%$ (IC95\%). O sorteio foi realizado de modo aleatório e estratificado, utilizando o aplicativo Excel, considerando as proporções de famílias e as subáreas de cada ESF. Portanto, foram sorteados 319 domicílios (138 na ESF-1 e 181 na ESF-2).

Foi elaborado um formulário com questões abertas e fechadas para coletar dados de identificação do domicílio cadastrado (código de cadastro na ESF), dos moradores (número de membros da família e suas idades) e do entrevistador (nome do pesquisador e data da visita). O formulário buscou ainda informações sobre consumo, origem das especialidades farmacêuticas identificadas (ESF e estabelecimentos públicos ou drogarias e farmácias comerciais), uso de medicamento na semana da entrevista, adequação do armazenamento segundo as condições de segurança e boas práticas de armazenamento $(15,16)$ e identificação e segurança do medicamento (nome do princípio ativo, prazo de validade, número de lote, bula e presença de embalagens). O formulário previa também um espaço para o registro do modo como os entrevistados usavam o medicamento e das orientações da prescrição médica. A utilização das recomendações da Organização Pan-Americana da Saúde (OPAS) e da regulamentação da Agência Nacional de Vigilância Sanitária (ANVISA) $(15,16)$ para determinar as boas práticas de armazenamento justifica-se pela ausência de normas específicas para o armazenamento domiciliar e porque as boas práticas devem ser as mesmas desde a produção, distribuição, varejo e uso do medicamento.

Os entrevistadores foram informados sobre os objetivos do estudo e treinados no preenchimento do formulário; em seguida, o instrumento foi validado em visita a 10 domicílios. Após pequenos ajustes, a coleta dos dados foi realizada nos meses de julho a outubro de 2008, pelos pesquisadores, sempre acompanhados pelos agentes comunitários de saúde.

Adotou-se como critério de inclusão, em cada domicílio sorteado, haver pelo menos um morador maior de 18 anos que aceitasse participar da entrevista, mediante assinatura do termo de consentimento livre e esclarecido, fornecendo informações sobre todos os moradores do domicílio sorteado. Foram excluídos do estudo os domicílios cujos moradores não atendessem os entrevistadores em até três tentativas de visita ou não aceitassem participar da entrevista.

Os dados coletados (existência de medicamentos, informações dos usuários de medicamento, descrição das condições de armazenamento, informações de identificação e segurança das especialidades farmacêuticas, ocorrência de automedicação, modo de uso do medicamento e informações da prescrição) foram processados em um banco de dados com dupla digitação, em aplicativo Excel, e tabulados na forma de frequência.

Para classificar as condições de armazenamento, estabeleceram-se quatro categorias: A) seguro e adequado; B) seguro e inadequado; C) inseguro e adequado; D) inseguro e inadequado. Foi considerado seguro o local fora do alcance de crianças, e adequado o local não exposto a luz, calor, umidade e sujeira e distante de animais, ralos, produtos de higiene, cosméticos e desinfetantes utilizados na limpeza doméstica.

A automedicação foi classificada como declarada quando o usuário do medicamento dizia ter tomado, naquela semana, algum medicamento por conta própria, ou observada, quando foi identificado em uso algum medicamento sob pres- 
crição vencida. Foi considerada vencida a prescrição com data de emissão superior a 30 dias, ou superior a 6 meses no caso de medicamentos de uso crônico.

O uso do medicamento foi considerado adequado sempre que o modo de uso declarado pelo usuário era o mesmo descrito na prescrição médica. O uso inadequado foi classificado em: desacordo posológico, quando a frequência ou dose mencionadas pelo usuário estavam diferentes da prescrição; e interrupção do tratamento, quando o usuário dizia não estar mais utilizando o medicamento prescrito.

O projeto foi aprovado pelo Comitê de Ética em Pesquisa do Hospital São Paulo da Universidade Federal de São Paulo (UNIFESP).

\section{RESULTADOS}

Foram sorteados 319 domicílios, sendo que, em 39 desses, os moradores não aceitaram participar ou não foram encontrados após três tentativas de visita. Os 280 domicílios (121 da ESF-1 e 159 da ESF-2) estudados representaram 994 indivíduos cadastrados nas ESF, sendo que $374 / 994(37,6 \%)$ tinham idade entre 18 e 40 anos.

Foram encontrados estoques de medicamentos em 255 domicílios (91,1\%). Em $217(77,5 \%)$, havia pelo menos um indivíduo fazendo uso de medicamentos, para um total de 350/994 indivíduos $(35,2 \%)$. A utilização mais frequente foi em crianças (entre 0 e 6 anos) e em maiores de 41 anos, principalmente nos idosos (acima de 60 anos) (figura 1).

Observou-se, em alguns domicílios, que o armazenamento de medicamentos era feito em mais de um local, havendo geralmente um local para medicamentos em uso e outro local para as sobras de antigos tratamentos. Em 212 casos $(65,1 \%)$, o local de armazenamento foi a cozinha. Apenas um em cada quatro locais foi considerado seguro e adequado (tabela 1).

Em 263 domicílios $(84,3 \%)$ houve falta de identificação e segurança nos medicamentos armazenados. Foram avaliadas 2578 especialidades farmacêuticas, das quais $2059(79,9 \%)$ apresentavam algum problema. Em média, os domicílios tinham 10,9 medicamentos estocados. A tabela 2 mostra os problemas observados nas especialidades farmacêuticas armazenadas nos domicílios.

Em 103 domicílios (36,8\%) havia pelo menos uma ocorrência de medicamentos

FIGURA 1. Distribuição dos indivíduos cadastrados na estratégia saúde da família segundo faixa etária e uso de medicamentos em um município do Estado de São Paulo, Brasil, 2008

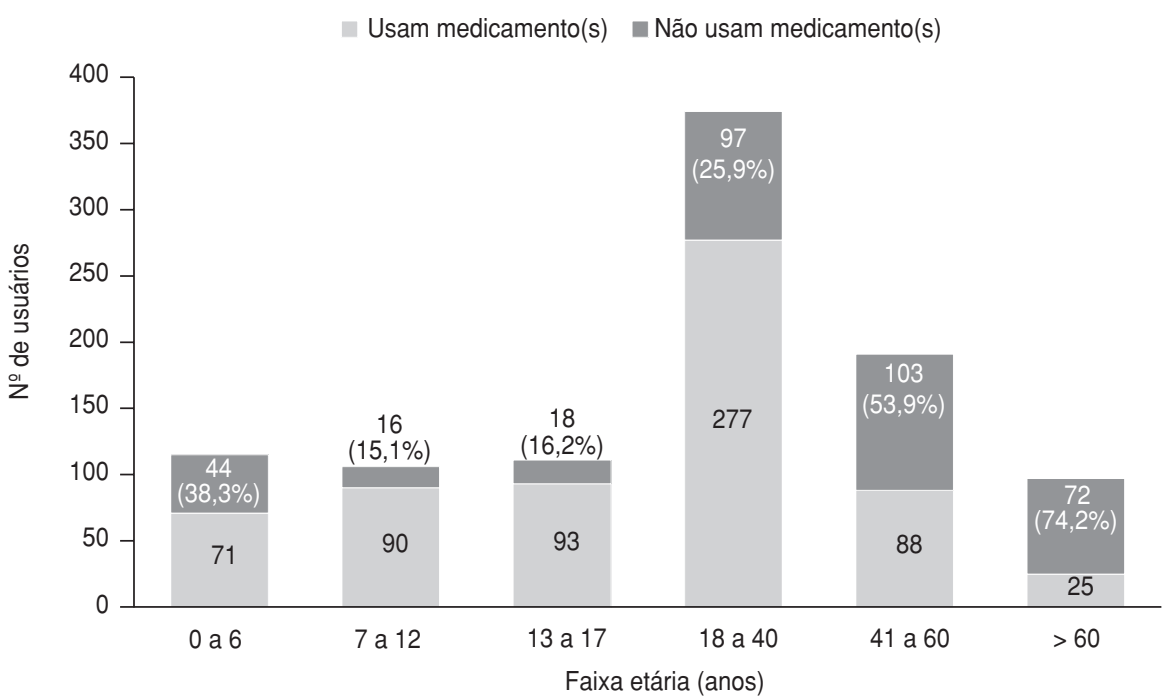

TABELA 1. Locais de armazenamento de medicamentos em domicílios assistidos pela estratégia saúde da família em um município do Estado de São Paulo, Brasil, 2008a

\begin{tabular}{|c|c|c|c|c|c|c|c|c|c|c|}
\hline \multirow[b]{2}{*}{ Local } & \multicolumn{2}{|c|}{$\begin{array}{c}\text { Seguro/ } \\
\text { adequado }\end{array}$} & \multicolumn{2}{|c|}{$\begin{array}{c}\text { Seguro/ } \\
\text { inadequado }\end{array}$} & \multicolumn{2}{|c|}{$\begin{array}{l}\text { Inseguro/ } \\
\text { adequado }\end{array}$} & \multicolumn{2}{|c|}{$\begin{array}{c}\text { Inseguro/ } \\
\text { inadequado }\end{array}$} & \multicolumn{2}{|c|}{ Total } \\
\hline & No. & $\%$ & No. & $\%$ & No. & $\%$ & No. & $\%$ & No. & $\%$ \\
\hline Cozinha & 47 & 14,4 & 62 & 19,0 & 13 & 4,0 & 90 & 27,6 & 212 & 65,1 \\
\hline Quarto & 25 & 7,6 & 17 & 5,2 & 11 & 3,4 & 38 & 11,6 & 91 & 27,9 \\
\hline Sala & 5 & 1,6 & 10 & 3,1 & 0 & 0,0 & 4 & 1,3 & 19 & 5,8 \\
\hline Banheiro & 2 & 0,6 & 0 & 0,0 & 0 & 0,0 & 2 & 0,6 & 4 & 1,2 \\
\hline Total & 79 & 24,2 & 89 & 27,3 & 24 & 7,4 & 134 & 41,1 & 326 & 100,0 \\
\hline
\end{tabular}

a Seguro = não acessível a crianças. Adequado: local não exposto a luz, calor ou umidade, produtos de higiene, cosméticos ou domissanitários, sujeira ou animais.

TABELA 2. Problemas de segurança e identificação das especialidades farmacêuticas armazenadas nos domicílios cadastrados na estratégia saúde da família em um município do Estado de São Paulo, Brasil, 2008

\begin{tabular}{lrrrrr}
\hline & \multicolumn{2}{c}{$\begin{array}{c}\text { Especialidades } \\
\text { farmacêuticas }\end{array}$} & & \multicolumn{2}{c}{ Domicílios } \\
\cline { 5 - 6 } \multicolumn{1}{c}{ Problema } & No. & $\%$ & & No. & $\%$ \\
\hline Ausência de bulas & 1927 & 74,7 & & 237 & 92,9 \\
Ausência de embalagem secundária & 1644 & 63,8 & & 232 & 90,9 \\
Especialidade com validade vencida & 359 & 13,9 & & 108 & 42,3 \\
Especialidade com lote ilegível & 187 & 7,3 & & 90 & 35,3 \\
Especialidade sem prazo de validade & 132 & 5,1 & & 78 & 30,6 \\
Especialidade com nome ilegível & 37 & 1,4 & & 30 & 11,7 \\
Sobra de antibióticos & 148 & 5,7 & & 77 & 30,2 \\
Sobra de medicamentos psicotrópicos e entorpecentes & 25 & 0,9 & & 16 & 6,3 \\
Ausência de embalagem primária & 1 & 0,04 & & 1 & 0,4 \\
$\quad$ Total & 2578 & 100,0 & & 100,0 \\
\hline
\end{tabular}

a Foi observado mais de um problema por especialidade farmacêutica avaliada.

com princípios ativos repetidos, totalizando 142 ocorrências. São exemplos dessa situação o princípio ativo dipirona, encontrado na denominação genérica e em apresentações comerciais,
Novalgina ${ }^{\circledR}$ e Anador ${ }^{\circledR}$; e o anti-inflamatório diclofenaco de sódio, também encontrado na denominação genérica e com nome comercial Biofenac ${ }^{\circledR}$. Dos medicamentos com princípios ativos repeti- 
dos, $83(58,5 \%)$ eram analgésicos e anti-inflamatórios. A dipirona foi o medicamento isento de prescrição mais recorrente (53 casos, 37,3\%), seguida dos anti-hipertensivos (13 casos, 9,2\%) e dos antibióticos (9 casos, 6,3\%).

Os $179(63,9 \%)$ entrevistados que utilizavam medicamentos declararam o modo de uso. Desses, $24(13,4 \%)$ se automedicavam, sendo que apenas um dos medicamentos utilizados na automedicação era isento de prescrição. Cinco dos medicamentos sob prescrição tinham receita vencida. Os demais medicamentos exigiam prescrição, mas os usuários não a tinham. Os medicamentos utilizados na automedicação foram analgésicos, anti-inflamatórios não esteroidais, antiulcerosos, antitérmicos, antiespasmódicos, relaxantes musculares e diuréticos tiazídicos. A aquisição de medicamentos para automedicação ocorreu principalmente em farmácias e drogarias (19 casos, ou 79,2\%) sem apresentação da prescrição médica; os medicamentos para automedicação adquiridos na ESF foram por meio de prescrição médica, porém no momento da entrevista a prescrição estava vencida.

Em apenas $44(24,6 \%)$ casos foi possível a comparação do modo declarado de uso dos medicamentos com as prescrições, porque os demais não as tinham. Desses, em $21(47,7 \%)$ foi identificado uso em desacordo com o modo prescrito, sendo 15 por diferenças posológicas e seis por interrupção do tratamento.

A mediana de medicamentos para os usuários que faziam uso incorreto foi de $5( \pm 1,8)$ medicamentos, sendo frequentes os anti-hipertensivos, hipoglicemiantes orais, insulina, anti-inflamatórios e antirreumáticos, diuréticos, antidepressivos e ansiolíticos, analgésicos, medicamentos para tratamento da úlcera péptica e antibióticos. Já entre os usuários que faziam uso de forma adequada, a mediana foi de $2( \pm 1,0)$, e as classes terapêuticas mais comumente utilizadas foram antihipertensivos, diuréticos tiazídicos, hipoglicemiantes orais e analgésicos.

\section{DISCUSSÃO}

Os dados do presente estudo evidenciam o acesso a medicamentos pela população estudada, como preconizado pela política nacional de medicamentos no propósito de promover o uso racional de medicamentos (1). No entanto, nem sempre o acesso garante o uso correto: nossos resultados mostram que um de cada quatro usuários entrevistados fazia uso inadequado de medicamentos.

Estudos apontam que a automedicação varia de 24,9 a $76,1 \%(4,11,17)$. Também indicam que a automedicação ocorre por meio do compartilhamento familiar, utilização de sobras de medicamentos, aquisição de medicamento sob prescrição sem apresentação do receituário ou com receituários antigos (18). Geralmente, a automedicação ocorre com os medicamentos das classes dos analgésicos, descongestionantes nasais, antiinflamatórios/antirreumáticos e antiinfecciosos de uso sistêmico (5).

A aquisição de medicamentos para automedicação ocorreu principalmente em farmácias e drogarias, como relatado pelos entrevistados, pois os medicamentos adquiridos nas ESF ou outras unidades de saúde pública sempre requerem uma prescrição médica. A prática de vender sem prescrição induz o consumo desnecessário de medicamentos e o uso sem diagnóstico, evidenciando ainda que as farmácias funcionam mais como comércio de medicamentos do que como prestadores de serviços, o que contraria a política nacional da assistência farmacêutica (19).

A comparação entre o modo de uso relatado e as orientações descritas nas prescrições médicas só foi possível neste estudo em 44 casos, pois os demais não possuíam a prescrição médica no momento da entrevista. Por si só, a ausência de prescrição pode motivar o uso inadequado do medicamento. A ausência da prescrição médica, assim como a ilegibilidade ou a falta de informações, são fatores que contribuem para o uso inadequado dos medicamentos e para a não adesão ao tratamento (20). Além disso, a ausência da prescrição prejudica a compreensão do tratamento e da doença e a relação entre paciente e prescritor, e compromete o uso concomitante de vários medicamentos e os esquemas posológicos complexos (21).

O desacordo posológico observado pode levar a doses subterapêuticas ou ainda a doses muito altas, podendo causar ineficácia do tratamento ou intoxicações e caracterizando, dessa forma, segundo a classificação do III Consenso de Granada, um resultado negativo associado à medicação (9). Além disso, os desacordos posológicos, somados à falta de acesso aos medicamentos, estão entre os responsáveis pelos elevados índices de morbimortalidade relacionada a medicamentos (2).

Observou-se que os usuários de medicamentos com inadequações no uso utilizavam mais medicamentos do que aqueles sem inadequações. Isso indica que, quanto maior o número de medicamentos em uso, maior a probabilidade de descumprir o tratamento, por confusão seja das doses ou das frequências de administração dos medicamentos - o que aumenta, consequentemente, a probabilidade de internações hospitalares por reações adversas a medicamentos (12).

Os dados alertam quanto à necessidade de uma maior atenção à população que utiliza medicamentos antidepressivos, ansiolíticos e antibióticos, pois foram essas as classes terapêuticas predominantes entre os indivíduos que apresentaram inadequações de uso. No caso dos antidepressivos e ansiolíticos, os erros no modo de uso podem ser explicados pelos próprios efeitos adversos inerentes à classe terapêutica, tais como prejuízo de atenção, memória e aprendizagem $(22,23)$. Quando utilizados sem o diagnóstico, a indicação e a orientação de um profissional da saúde, ou ainda de forma inadequada, os medicamentos psicotrópicos podem causar dependência $(22,23)$. Já as inadequações no uso dos antibióticos também devem ser prevenidas, pois seu uso indiscriminado pode manter ou agravar a doença infecciosa e aumentar a resistência bacteriana e os gastos com hospitalizações $(24,25)$. Ademais, os medicamentos psicotrópicos e entorpecentes e os antibióticos também estavam presentes dentre as sobras de medicamentos, favorecendo, assim, as trocas entre os indivíduos da residência e a possibilidade de intoxicação acidental.

Os idosos eram os que mais utilizavam medicamentos $(74,2 \%)$. Sugere-se uma atenção maior a essa faixa etária, por apresentar alterações fisiológicas e cognitivas inerentes à idade e geralmente ser a mais polimedicada $(26,27)$. Dessa forma, os idosos estão mais propensos a ter problemas relacionados ao uso do medicamento, tais como erros de dosagem, troca de medicamentos, duplicidade de princípios ativos, interações, reações adversas e intoxicações, entre outros, necessitando de ajustes posológicos, orientações e acompanhamento farmacoterapêutico.

Os locais de armazenamento, em sua maioria, foram classificados como inse- 
guros e inadequados: inadequados por estarem os medicamentos expostos ao sol, umidade, calor, sujeira, animais domésticos ou substâncias como perfumes, cosméticos e desinfetantes utilizados na limpeza; e inseguros por estarem ao alcance de crianças. Dado semelhante foi observado por Schenkel et al. (28).

Sabe-se que as crianças são as principais vítimas de ingestão acidental de medicamento e de intoxicações (7). Portanto, cabe aos serviços de saúde promover intervenções educativas, tais como distribuição de material informativo às famílias assistidas pela ESF e orientação ao cuidador responsável pela criança quanto à forma adequada de guarda dos medicamentos - em lugar alto, com portas trancadas ou de difícil abertura.

Já o armazenamento inadequado leva ao comprometimento da qualidade e da efetividade dos medicamentos, pois favorece a degradação dos princípios ativos, diminuindo sua eficácia ou promovendo a formação de outras substâncias tóxicas, o que também compromete a estabilidade dos medicamentos e causa risco de intoxicação.

Na maioria dos domicílios, as especialidades farmacêuticas estavam sem data de validade, bula, número do lote ou nome do princípio ativo. A falta dessas informações leva ao uso de medicamentos vencidos, constituindo um risco à saúde do usuário, e ao uso incorreto, pela confusão de medicamentos com características semelhantes. Além disso, em casos de inefetividade terapêutica, intoxicações e reações adversas, a ausência dessas informações inviabiliza a identificação do medicamento utilizado e do seu fabricante.

Os medicamentos armazenados fora de suas embalagens secundárias tornamse um risco de segurança, pois não estão protegidos e sua rastreabilidade está comprometida, ou seja: se houver alguma reação adversa ao medicamento, ineficácia terapêutica ou desvio de qualidade do medicamento, este não poderá ser identificado quanto ao nome, princípio ativo, prazo de validade ou lote. $\mathrm{Ob}-$ servou-se que é uma prática daqueles que utilizam medicamentos guardar os mesmos apenas na embalagem primária (90,9\%). Isso mostra a urgência de se implantar um sistema que disponibilize os medicamentos em embalagens fracionáveis, cuja embalagem primária apresente o nome do princípio ativo, prazo de vali- dade, número do lote e fabricante (29), evitando trocas e confusão entre medicamentos semelhantes e colaborando para o uso racional de medicamentos, principalmente dos isentos de prescrição, que são os mais comumente estocados. Ademais, essa medida reduziria o desperdício e o número de especialidades estocadas, facilitando o manejo (28).

A maioria das especialidades farmacêuticas não continha suas respectivas bulas. Sabe-se que, geralmente, os medicamentos distribuídos pelo Sistema Único de Saúde (SUS) são fabricados por laboratórios oficiais e que esses não fornecem as bulas. Entende-se que essa prática compromete o acesso à informação adequada dos medicamentos pelos que os utilizam, podendo comprometer $\mathrm{o}$ uso correto.

A ocorrência de medicamentos com princípios ativos repetidos pode levar à intoxicação se houver administração concomitante. Observou-se que os medicamentos frequentemente repetidos eram os analgésicos, que são isentos de prescrição; no entanto, não são isentos de riscos e de necessidade de orientação para garantir seu uso adequado. Em seguida estavam os anti-inflamatórios, que, apesar de exigir prescrição, são adquiridos em farmácias e drogarias sem a indicação de um médico ou dentista por meio de um receituário.

Os presentes resultados mostram que o exercício farmacêutico no âmbito do cuidado primário e da ESF permitiria a identificação, correção e prevenção de vários problemas, garantindo o recebimento da terapia medicamentosa da forma adequada, efetiva, segura e cômoda pelo paciente. A atuação do farmacêutico que mais atende aos princípios da ESF - prevenção de doenças, promoção e recuperação da saúde - é a atenção farmacêutica, como define o Consenso Brasileiro de Atenção Farmacêutica - um modelo de prática que compreende "atitudes, valores éticos, comportamentos, habilidades, compromissos e corresponsabilidades na prevenção de doenças, promoção e recuperação da saúde, de forma integrada à equipe de saúde" (grifo nosso). Essa atenção farmacêutica envolve interação direta entre o farmacêutico e o usuário do medicamento e entre o farmacêutico e a comunidade, e respeita as especificidades biopsicossociais do sujeito a partir da ótica da integralidade das ações de saúde (30).
Uma das limitações deste estudo foi a amostragem utilizada. Apesar do sorteio aleatório e estratificado, foram excluídos os moradores que não atenderam os entrevistadores depois de três tentativas de visita ou não aceitaram participar da entrevista. Isso traz um risco de viés de seleção, visto que a recusa em participar da pesquisa pode indicar embaraço do morador perante a temática abordada.

Outra limitação foi a avaliação do uso dos medicamentos, pois, para estimar a automedicação, consideramos a informação declarada pelo usuário, já que apenas 44 dispunham da prescrição médica. Apenas nesses casos se avaliou o uso quanto à posologia e duração do tratamento; outros parâmetros não puderam ser avaliados, pois mesmo nas prescrições médicas faltavam algumas informações imprescindíveis para o uso correto dos medicamentos.

\section{Conclusões}

Os dados obtidos indicam que na maioria dos domicílios havia pelo menos um usuário de medicamentos. Além disso, na maioria dos domicílios os medicamentos eram estocados de forma inapropriada, sinalizando a necessidade de disponibilização em embalagens fracionais para diminuir as sobras que geram os estoques domésticos.

A automedicação é uma prática preocupante por ser realizada de forma inadequada e abusiva e, na maioria das vezes, com medicamentos sujeitos a prescrição médica, incluindo medicamentos de controle especial. Os desacordos posológicos e as interrupções de tratamento por nós identificados possivelmente estão associados a resultados negativos associados à medicação, em função de doses baixas, ou a reações adversas, podendo causar intoxicações por doses elevadas e não adesão terapêutica. Além disso, as inadequações de uso foram mais frequentes entre os usuários polimedicados, indicando a necessidade de um maior cuidado $\mathrm{e}$ atenção a esses pacientes pelos profissionais de saúde.

$\mathrm{O}$ acesso a medicamentos é um importante passo a ser cumprido. No entanto, segundo as diretrizes da política nacional de medicamentos, para que seu uso seja adequado, ainda se faz necessário um acompanhamento farmacoterapêutico, por exemplo, com a participação do profissional farmacêutico. Na ESF a 
atenção farmacêutica poderia identificar, corrigir e prevenir os problemas do uso incorreto dos medicamentos, a fim de promover seu uso racional e a melhoria do estado de saúde da comunidade. Essa atenção farmacêutica deveria, finalmente, ser apoiada por toda a equipe multiprofissional: o médico, que, ao prescrever, deve dar orientações sobre o uso e armazenamento dos remédios; a enfermagem, que frequentemente complementa e reforça essas orientações; e os agentes de saúde, muitas vezes mais próximos dos domicílios, podendo, assim, observar e relatar a prática das comunidades.
Agradecimentos. À equipe multidisciplinar das unidades de estratégia saúde da família por colaborar e permitir a realização deste estudo. À Pró-Reitoria de Extensão da Universidade Estadual Paulista (UNESP) e à Fundação para o Desenvolvimento da UNESP (processo 11 709/09 e 43/10-DFP) pelo apoio financeiro.

\section{REFERÊNCIAS}

1. Brasil, Ministério da Saúde, Secretaria de Políticas de Saúde. Política nacional de medicamentos. Rev Saude Publica. 2000;34(2):206-9.

2. Organización Mundial de la Salud. Perspectivas políticas sobre medicamentos de la OMS. Promoción del uso racional de medicamentos: componentes centrales. Disponível em: http://apps.who.int/medicinedocs/pdf/s48 74s/s4874s.pdf. Acessado em 11 de fevereiro de 2010.

3. Brasil. Portaria $3961 / 1998$. Disponível em: http://www.cff.org.br/userfiles / file/por tarias/3916_gm.pdf. Acessado em 11 de fevereiro de 2010.

4. Aquino DS. Por que o uso racional de medicamentos deve ser uma prioridade? Cienc Saude Coletiva. 2008;13(supl 0):733-6.

5. Arrais PSD, Coelho HLL, Batista MCDS, Carvalho ML, Righi RE, Arnau JM. Perfil da automedicação no Brasil. Rev Saude Publica. 1997;31(1):71-7.

6. Margonato FB, Thomson Z, Paoliello MMB. Determinantes nas intoxicações medicamentosas agudas na zona urbana de um município do Sul do Brasil. Cad Saude Publica. 2008;24(2):333-41.

7. Brasil, Ministério da Saúde, Sistema Nacional de Informações Tóxico-Farmacológicas SINITOX. Registro de intoxicações. Dados nacionais. Casos registrados de intoxicação e/ ou envenenamento. 2009. Disponível em: http://www.fiocruz.br/sinitox_novo/cgi/ cgilua.exe/sys/start.htm?sid=8. Acessado em 20 de janeiro de 2010.

8. Bortolon PC, Karnikowski MGO, Assis M. Automedicação versus indicação farmacêutica: o profissional de farmácia na atenção primária à saúde do idoso. Rev APS. 2007; 10(2):200-9.

9. Cipolle RJ, Strand LM, Morley PC. Pharmaceutical care practice: the clinician's guide. $2^{\text {a }}$ ed. Columbus: McGraw-Hill; 2004.

10. Comité de Consenso: Grupo de Investigación en Atención Farmacéutica (CTS-131), Universidad de Granada (España), Grupo de Investigación en Farmacología (CTS-164), Universidad de Granada (España), Fundación Pharmaceutical Care España, Sociedad Española de Farmacia Comunitaria (SEFaC). Tercer Consenso de Granada sobre Problemas Relacionados con Medicamentos (PRM) y Resultados Negativos Asociados a la Medicación (RNM). Ars Pharm. 2007;48(1):5-17.
11. Vilarino JF, Soares IC, Silveira CM, Rödel APP, Bortoli R, Lemos RR. Perfil da automedicação em município do Sul do Brasil. Rev Saude Publica. 1998;32(1):43-49.

12. Mastroianni PC, Varallo FR, Barg MS, Noto AR, Galduróz JCF. Contribuição do uso de medicamentos para a admissão hospitalar. Braz J Pharm Sci. 2009;45(1):163-70.

13. Zargarzadeh $\mathrm{AH}$, Emani $\mathrm{MH}$, Hosseini $\mathrm{F}$. Drug-related hospital admissions in a generic pharmaceutical system. Clin Exp Pharmacol Physiol. 2007;34:494-8.

14. Instituto Brasileiro de Geografia e Estatística. IBGE Cidades, 2007. Disponível em: http:// www.ibge.gov.br/cidadesat/topwindow.ht $\mathrm{m}$ ?1. Acessado em 11 de fevereiro de 2010.

15. Aguilar NG, Bittner MR. Guía para el desarrollo de servicios farmacêuticos hospitalarios: selección y formulario de medicamentos. Programa de Medicamentos Esenciales y Tecnología (HSE). República Dominicana: Organización Panamericana de la Salud; 1997. Disponível em: http://www.opas.org.br/ medicamentos/temas_documentos_detalhe. $\mathrm{cfm} ? \mathrm{id}=39$ \&iddoc $=407$. Acessado em $11 \mathrm{de}$ fevereiro de 2010.

16. Brasil. Resolução RDC 210/2003. Disponível em: http://www.anvisa.gov.br/legis/resol/ 2003/rdc/210_03rdc.pdf. Acessado em 11 de fevereiro de 2010.

17. Vosgerau MZS, Soares DA, Souza RKT. Automedicação entre adultos na área de abrangência de uma Unidade de Saúde da Família. Lat Am J Pharm. 2008;27(6):831-8.

18. Loyola Filho AI, Uchoa E, Guerra HL, Firmo JOA, Lima-Costa MF. Prevalência e fatores associados à automedicação: resultados do projeto Bambuí. Rev Saude Publica. 2002; 36(1):55-62.

19. Brasil, Ministério da Saúde. 1ํㅡ Conferência Nacional de Medicamentos e Assistência Farmacêutica: relatório final. Efetivando o acesso, a qualidade e a humanização na assistência farmacêutica, com controle social. Disponível em: http://conselho.saude.gov. $\mathrm{br} /$ biblioteca/Relatorios/confer_nacional_de $\% 20$ medicamentos.pdf Acessado em $11^{1}$ de fevereiro de 2010.

20. Mastroianni PC. Análise dos aspectos legais das prescrições de medicamentos. Rev Cienc Farm Basica Apl. 2009;30(2):45-8.

21. Becker MH. Patient adherence to prescribed therapies. Med Care. 1985;23(5):539-55.
22. Andrade MF, Andrade RCG, Santos V. Prescrição de psicotrópicos: avaliação das informações contidas em receitas e notificações. Rev Bras Cienc Farm. 2004;40(4):471-9.

23. Chetley A. Health Action International: Psychotropics - tales of dependence. 10A. Amsterdã: HAI; 1993.

24. Abrantes PM, Magalhães SMS, Acúrcio FA, Sakurai E. Avaliação da qualidade das prescrições de antimicrobianos dispensadas em unidades públicas de saúde de Belo Horizonte, Minas Gerais, Brasil, 2002. Cad Saude Publica. 2007;23(1):95-104.

25. World Health Organization. Interventions and strategies to improve the use of antimicrobials in developing countries: Drug Management Program. Disponível em: http:// www.who.int/csr/resources/publications/ drugresist/WHO_CDS_CSR_DRS_2001_9/ en/. Acessado em 11 de fevereiro de 2010.

26. Marin MJS, Cecílio LCO, Perez AEWUF, Santella F, Silva CBA, Gonçalves Filho JR, et al. Caracterização do uso de medicamentos entre idosos de uma unidade do Programa Saúde da Família. Cad Saude Publica. 2008;24(7):1545-55.

27. Rozenfeld S. Prevalência, fatores associados e mau uso de medicamentos entre os idosos: uma revisão. Cad Saude Publica. 2003;19(3): 717-24.

28. Schenkel EP, Fernándes LC, Mengue SS. Como são armazenados os medicamentos nos domicílios? Acta Farm Bonaerense. 2004;24 (2):266-70.

29. Brasil. Resolução RDC 80/2006. Disponível em: http://www.crfsp.org.br/joomla/index. php?option $=$ com_content\&view $=$ article\&id $=$ 689:resolucao-rdc-no-80-de-11-de-maio-de2006\&catid $=113$ :legislacao\&Itemid $=58$. Acessado em 11 de fevereiro de 2010.

30. Organização Panamericana da Saúde. Atenção farmacêutica no Brasil: trilhando caminhos - relatório 2001-2002. Disponível em: http://www.opas.org.br/medicamentos/ temas documentos detalhe.cfm?id=43\&iddo $c=245$. Acessado em 11 de fevereiro de 2010.

Manuscrito recebido em 4 de julho de 2010. Aceito em versão revisada em 21 de outubro de 2010. 
ABSTRACT Objective. To identify families served by the family health strategy (FHS) storing medicines at home, to evaluate storage conditions, and to investigate medicine use practices.

Household storage and use of medications in a population served by the family health strategy in Brazil

Key words
Methods. The study was conducted in a municipality in the state of São Paulo with two FHS units serving 1867 households. The sample was selected by means of stratified random sampling. Data collection was conducted through semistructured interviews from July to October 2008.

Results. One resident was interviewed in each of the 280 households visited. Medicines were found in 255 households (91.1\%). Of 326 storage locations, $217(75.8 \%)$ were inadequate (easily accessible to children or exposed to moisture, light). Of the 2578 medicines identified, 2059 medicines (79.9\%) in $236(84.3 \%)$ households had safety or identification problems. Of the 280 respondents, $179(63.9 \%)$ used medications. Of these, 24 were self-medicating, only one with an over-the-counter drug. Only 44 users had the prescription for their medication, and 21 did not follow the prescription in terms of dosage or had interrupted the treatment.

Conclusions. Non-adherence to recommended treatment can lead to negative outcomes, such as inefficiency (using dosages lower than prescribed), poisoning (using dosages higher than prescribed), and other adverse reactions.

Pharmaceutical services; Family Health Program; drug utilization; drug storage; self medication; Brazil. 